\title{
Treatment Results of Local Steroid Administration in Breast-Feeding Women with Idiopathic Granulomatous Mastitis
}

\author{
Osman Toktas ${ }^{1 *}$ and Nursen Toprak ${ }^{2}$ \\ ${ }^{1}$ Department of General surgery, Yuzuncu Yil University, Turkey \\ ${ }^{2}$ Department of radiology, Yuzuncu Yil University, Turke
}

Submission: February 10, 2021; Published: February 16, 2021

*Corresponding author: Osman Toktas, Department of General surgery, Yuzuncu Yil University, Turkey

\begin{abstract}
Introduction: Idiopathic granulomatous mastitis (IGM) is an inflammatory and chronic benign disease of the breast. The choice of treatment for IGM can prove exceedingly difficult. For one, some treatment modalities cannot be used in breast-feeding patients. At the same time, a misdiagnosis of IGM can overlook puerperal mastitis, resulting in an increased need for surgery. For a breast-feeding patient, there is strong basis to believe that, when IGM is properly diagnosed, the preferred treatment rests with long-term oral steroid therapy. In this study, to our knowledge the first of its kind, we assess the results of local steroid therapy in breast-feeding women with IGM.

Methods: We analyzed pregnant women with IGM between June 2017 and September 2019. The six breast-feeding women under study were treated by using intralesional steroid injection and topical steroid. Treatment response was evaluated both clinically and radiologically, at the end of two weeks and, once more, at the end of one month.

Result: The median age of the patients was 26 years. The mean duration of complaints was 4.3 months. The median number of children was 2 , and the mean breast-feeding time was 41 months. The predominant complaints at onset were a breast mass or local pain and inflammation in all patients. Diagnosis was made with incisional biopsy in all patients who undergo abscess drainage. Four patients (66.6\%) achieved a complete response, and two patients (33.4\%) responded only partially after the first course of treatment. A second course of treatment was given the patient with partial response. Five patients achieved a complete response at the end of the second course of treatment. One patient had a lumpectomy. The mean follow-up time was 15.1 months. During the follow-up period, one patient suffered recurrence at 9 months, and she received a third course of treatment. Topical and systemic side effects of corticosteroids were not seen in any patient.
\end{abstract}

Conclusion: When IGM is confused with periductal mastitis during the breast-feeding period, accurate diagnosis is delayed, and the need for surgery increases. Use of local corticosteroid in the treatment of breast-feeding women with IGM is effective in terms of response to treatment, duration of treatment, need for surgery, and side effects.

keywords: Ldiopathic granulomatous mastitis; Granulomatous mastitis in breast-feeding women; Steroid injection; Topical steroid

Abbreviations: IGM: Idiopathic Granulomatous Mastitis; PAS: Periodic-Acid-Schiff; EZN: Ehrlich-Ziehl-Neelsen; USG: Ultrasonography; LGM: Lobular Granulomatous Mastitis

\section{Introduction}

Idiopathic granulomatous mastitis (IGM) is a benign and chronic inflammatory disorder of the breast. Histologically, IGM is characterized by non-caseating, non-vascular granulomatous inflammatory changes in breast lobules. Although there is no consensus on optimal treatment, various medical and surgical treatment modalities have been used to treat IGM [1-5]. The most common treatment modalities are corticosteroid and surgery
[6-8]. Surgery has cosmetic and healing challenges for breast-feeding patients; on the other hand, in some patients, corticosteroids can have side effects and must be used for a long period to get an adequate response. In recent studies, local corticosteroids were found to be effective, better tolerated, and having fewer side effects [1,9-11]. In our study, we assess intralesional steroid injection with concomitant topical steroids in the treatment of IGM in breast-feeding women. 


\section{Materials and Methods}

The data collected between June 2017 and September 2019 was analyzed retrospectively. Breast-feeding patients who visited the breast clinic with complaints of breast mass, breast pain, and local erythema were evaluated clinically and radiologically. Patients with suspected IGM underwent core needle and incisional biopsy according to their clinical presentation. The diagnosis of IGM was made histopathological by pathologists working in our Center.

In the histopathological diagnosis of each patient, infectious granulomatous mastitis agents were excluded with Gram, Periodic-Acid-Schiff (PAS), Ehrlich-Ziehl-Neelsen (EZN) and Giemsa stains. Breast-feeding women who were diagnosed with IGM and received treatment with local corticosteroids with at least 12 months of follow-up were included in the study. For patients treated with local steroids, protocol included an intralesional 40mg methylprednisolone (Prednol-L 20mg amp.; Mustafa Nevzat Pharmaceuticals, Istanbul, Turkey) injection into the breast skin affected by mastitis and administration of topical 0.125\% prednisolone (Prednol-a 30gr pomad; Mustafa Nevzat Pharmaceuticals, Istanbul, Turkey) twice a day, on alternate days, for 4 weeks. Patients who have diffuse disease were injected at different points, at intervals of $5 \mathrm{~cm}$.

Patients with recurrent disease and patients for whom use of corticosteroids was contraindicated were excluded from the study. Patients were followed up in terms of treatment results. Treatment response was evaluated both clinically and radiologically at the end of two weeks and, again, at the end of six weeks. The response was categorized as "complete response," "partial response," "no response," or "worsening disease." Patients with "complete response" were assigned follow-up, while patients with "partial response," "no response," or "worsening disease" received a second and, if necessary, a third course of treatment, with monthly check-ups until resolution of the disease. Surgical treatment was a second option for all patients who clinically required or preferred surgery during follow-up.

\section{Statement of Ethics}

This study was approved by the local ethics committee of Van Yuzuncu Yil University, Turkey with the registration number of 2020/04-33.

\section{Result}

Six breast-feeding women were included in the study. While 4 patients were newly diagnosed, 2 patients had a previous history of abscess in the breast. For none of these patients was there a confirmed diagnosis of IGM. The disease localized in the left breast in five patients, and in the right breast in one patient. Mean age of the patients was 29 years (range 22-38). There was no comorbid medical situation in the medical history in any patients. The mean duration of complaints was 3 (range1-6) months. While the mean number of children was 2 (range 2-5), the mean breast-feeding time was 49.5 months (range 25-84). The predominant complaints at onset were a breast mass with local pain and inflammation in all patients. Abscess drainage was performed in all patients, and four of them had fistulation to the skin. Diagnosis was made with an incisional biopsy in all these patients. All patients had made prior use of antibiotics. The patients continued breast-feeding from the other breast during the treatment. While ultrasonography (USG) was used in all patients, USG plus MG was used in only one patient.

Table 1: Demographic, clinical characteristics of the patients.

\begin{tabular}{|c|c|c|}
\hline & & N (\% /range) \\
\hline Mean Age & & $29(22-38)$ \\
\hline Mean duration of complaints (month) & & $3(1-6)$ \\
\hline Mean number of children & & $2(2-5)$ \\
\hline Mean breast-feeding time (month) & Left & Right \\
\hline Localization & Breast mass with local pain and inflammation & $1(25-84)$ \\
\hline & Breast mass and pain without inflammation & $5(83.3)$ \\
\hline Main complaint at onset & Incisional & $1(16.7)$ \\
\hline Treatment response (first course) & Complete & $6(100)$ \\
\hline & Partial & $3(50)$ \\
\hline Treatment response (second course-3 patients) & Complete & $3(50)$ \\
\hline
\end{tabular}


Four patients $(66.6 \%)$ achieved a complete response, and two patients (33.4\%) achieved a partial response after the first course of treatment. A second course of treatment was given to the patient with partial response. Complete response was achieved by five patients at the end of the second course of treatment (Figures 1 \& 2). One patient underwent a lumpectomy because of insufficient response after the second dose and the patient did not want to continue treatment. One patient $(16.6 \%)$ had recurrence at 9 months during the follow-up period, and a third course was administered to this patient. The mean follow-up time was 15.1 months (range 12-21). Neither topical nor systemic side effects of corticosteroids were seen in any patient. Patients and their clinical characteristics are shown in the accompanying Table 1.

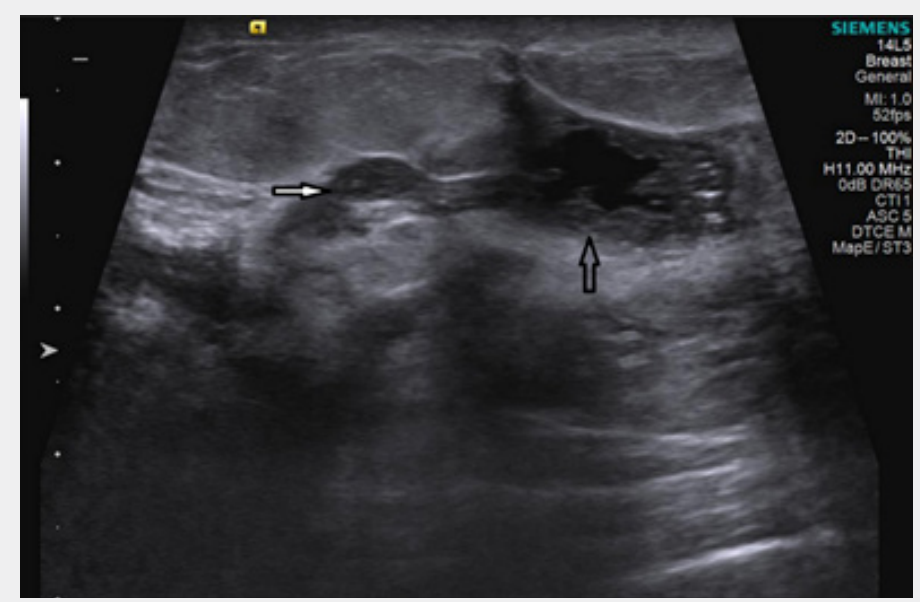

Figure 1a: 32 years-old women, the mass with tubular extensions (white arrow to right), and hypoechoic irregular margin (upper hollow arrow).



Figure 1b: Regression in the mass (upper hollow arrow) and tubular extensions (white right arrow) after treatment.

\section{Discussion}

Lobular granulomatous mastitis (LGM) or idiopathic granulomatous mastitis (IGM) that affects young or middle-aged women is a noncaseating chronic granulomatous lobules [12,13]. Although the specific etiology of IGM is not known, studies have shown that pathogenesis may be explained by nonspecific lobulitis due to lobule deformation [14,15]. Since its incidence is rare and its etiology uncertain, there is no clear path in the management and treatment of IGM. Treatment modalities such as observation, steroids, operative intervention, or immunosuppressant therapy (e.g., methotrexate) have been used, as the literature indicates [16]. 


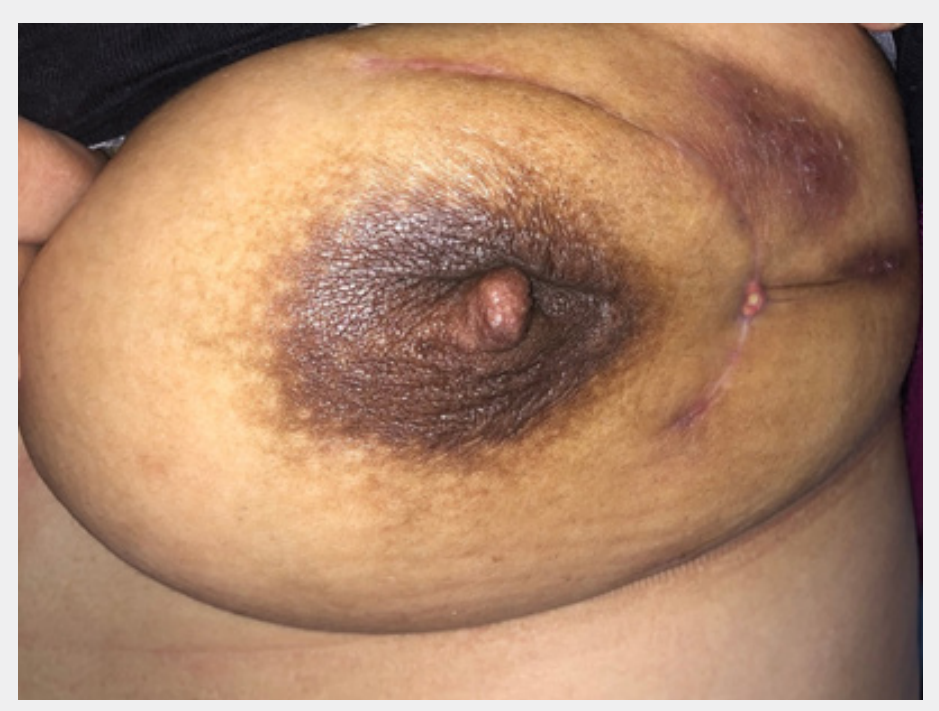

Figure 2a: The mass fulling left breast external quadrant with fistulas on the skin and drainage was performed due to abscess 3 weeks ago.

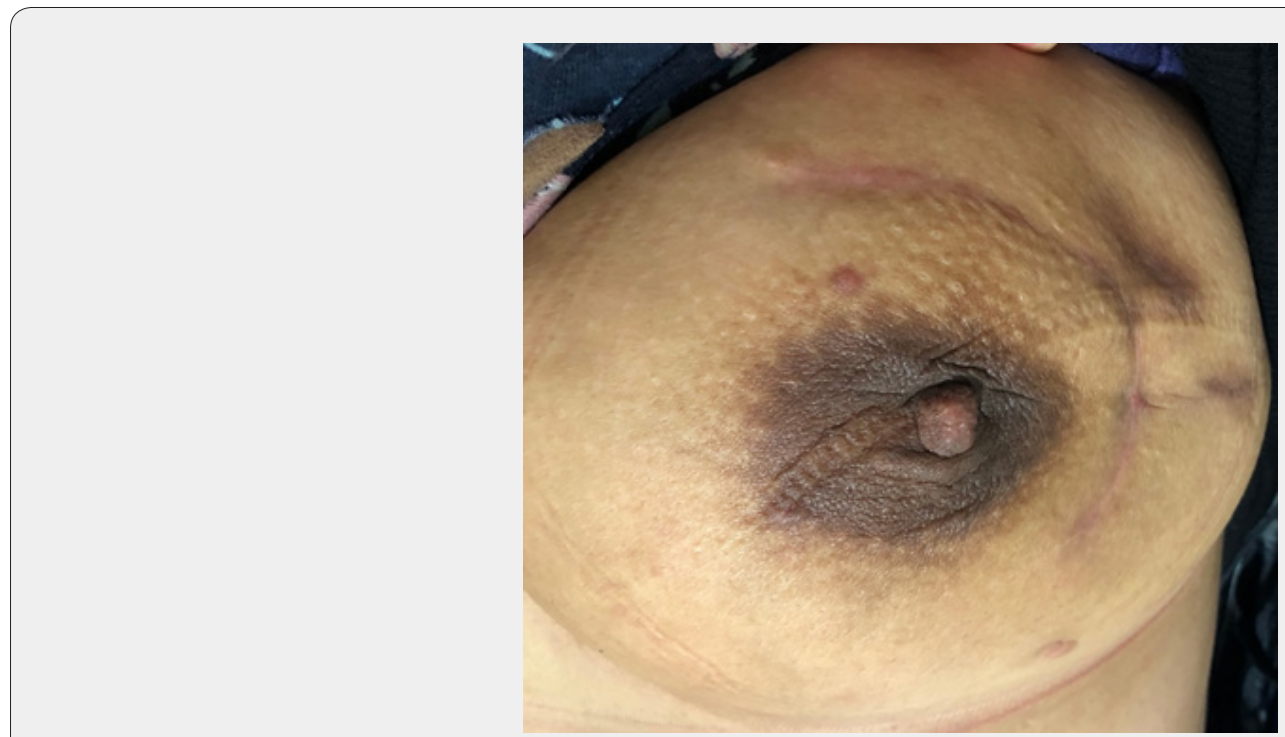

Figure $\mathbf{2 b}$ : After first course of treatment, regression was observed in the lesions in all quadrants.

While the literature contains many studies on the use of oral steroids and surgery, the effectiveness of local steroid use has been demonstrated in only a few current studies $[1,10,11]$. Because the number of breast-feeding cases with IGM is very low in the literature and local prednisone treatment has not been used in these patients before, we wanted to assess the effect of topical prednisone in breast-feeding women with IGM in what, to our knowledge, is the first study of this kind.

There are pros and cons to both surgery and conservative therapy with steroids [9] For instance, while limited excision can reduce recurrence, wide local excision may give poor cosmetic results. Because of a delay in diagnosis due to confusion with puerperal mastitis, or because there is a limited choice of medical treatment modalities during the breast-feeding period, surgery is used more frequently in these patients $[17,18]$. In our study, all patients had used antibiotics before diagnosis, and the mean duration of complaints was 3 months. Abscess drainage and incisional biopsy were done in all patients. Delay in diagnosis was the result of confusion with puerperal mastitis during breastfeeding.

Significant regression in breast mass and skin lesions may be achieved with systemic corticosteroids, but corticosteroids used in high dosage or over a long period of time can have side effects [19]. In our former study that compared the results of treatment with topical and systemic steroids, we observed that patients treated with topical steroids had a shorter duration of treatment, 
had fewer steroid-related side effects, had less need for surgery, and had lower recurrence rates. Use of topical steroid in the treatment of IGM has been assessed in other studies [1,11].

There is no consensus on IGM treatment for breast-feeding patients, and the treatment protocol is not clear. However, the most commonly used treatment modality is oral prednisone therapy. The duration of treatment with oral prednisone is longer, and patients' compliance with the treatment is difficult $[5,20]$. Kaviani et al. [4] In that study, symptoms started during breastfeeding in 27 patients (7.6\%), and these patients were treated with oral prednisone. In the study of Al-Khaffaf et al. [5], the mean recovery time was 43 weeks, and this period was longer than 60 weeks in patients with breast-feeding history. Goulabchand et al. [20] were able to achieve a complete response 6 months later in patients who started oral steroids (10mg daily of prednisone) during breast-feeding, and they recommend that these patients be treated with oral prednisone for 4 months. In the case reports of Goldberg et al. [21] prednisone was started postpartum and the breast improved 2 months later. Complete resolution of wounds came after 5 months.

In our study, we used only intralesional $40 \mathrm{mg}$ methylprednisolone and an administration of topical $0.125 \%$ prednisolone cream. At the end of 2 months, we had complete remission in five (83.3\%) patients. We found that treating patients with topical prednisone brings a host of advantages, among them stress reduction, increased patient compliance and a reduction in the cost of treating the disease. We did not see any side effects related to steroids in mother or baby. Patients showed no problems with breastfeeding. Moreover, we saw none of the unfortunate cosmetic results so common to repeated surgeries. Our patients compliance and satisfaction with this topical steroid treatment, in which only two drugs were used for a short time, were highly encouraging.

\section{Conclusion}

We think that the local corticosteroid treatment should be the first step in the treatment of breast-feeding women with IGM, given the more positive response seen in a relatively short time, the patient's high compliance with treatment, and the lower rates of side effects and recurrence.

\section{References}

1. Toktas O, Konca C, Trabulus DC, Soyder A, Koksal H, et al. (2020) A Novel First-Line Treatment Alternative for Noncomplicated İdiopathic Granulomatous Mastitis: Combined İntralesional Steroid İnjection with Topical Steroid Administration. Breast care pp. 30.

2. Han BK, Choe YH, Park JM, Moon WK, Ko YH, et al. (1999) Granulomatous mastitis: mammographic and sonographic appearances. AJR Am J Roentgenol 173(2): 317-320.

3. Ocal K, Dag A, Turkmenoglu O, Tuba K, Hakan S, et al. (2010) Granulomatous mastitis: clinical, pathological features, and management. Breast J 16(2): 176-182.
4. Kaviani A, Vasigh M, Omranipour R, Mahmoudzadeh H, Elahi A, et al. (2019) Idiopathic granulomatous mastitis: Looking for the most effective therapy with the least side effects according to the severity of the disease in 374 patients in Iran. Breast J 25(4): 672-677.

5. Al-Khaffaf B, Knox F, Bundred NJ (2008) Idiopathic granulomatous mastitis: a 25-year experience. J Am Coll Surg 206(2): 269-273.

6. Sheybani, F, Naderi HR, Gharib M, Sarvghad M, Mirfeizi Z, et al. (2016) Idiopathic granulomatous mastitis: Long-discussed but yet-to-beknown. Autoimmunity 49(4): 236-239.

7. Uysal E, Soran A, Sezgin E (2018) Granulomatous Mastitis Study Group. Factors related to recurrence of idiopathic granulomatous mastitis: what do we learn from a multicentre study? ANZ J Surg 88(6): 635-639.

8. Chirappapha P, Thaweepworadej P, Supsamutchai C, Biadul N, Lertsithichaia P, et al. (2018) Idiopathic granulomatous mastitis: A retrospective cohort study between 44 patients with different treatment modalities. Ann Med Surg (Lond) 36: 162-167.

9. Wang C, Lin Y, Zhou Y, Sun Q (2020) Novel Paradigm for Treating Idiopathic Granulomatous Mastitis. J Invest Surg 21: 1-2.

10. Altintoprak F, Kivilcim T, Yalkin O, Uzunoglu Y, Kahyaoglu Z, et al. (2015) Topical Steroids Are Effective in the Treatment of Idiopathic Granulomatous Mastitis. World J Surg 39(11): 2718-2723.

11. Alper F, Karadeniz E, Guven F, Çankaya BV, Özden V, et al. (2020) The evaluation of the efficacy of local steroid administration in idiopathic granulomatous mastitis: The preliminary results. Breast J 26(2): 309311.

12. Sheybani F, Sarvghad M, Naderi H, Gharib M (2015) Treatment for and clinical characteristics of granulomatous mastitis. Obstet Gynecol 125(4): 801-807.

13. Haddad M, Sheybani F, Arian M, Gharib M (2020) Methotrexate-based regimen as initial treatment of patients with idiopathic granulomatous mastitis. Breast J 26(2): 325-327.

14. John D, Daniela C, Samantha M, Chiu-Hsieh H, Morgan JB, et al. (2019) Re-evaluating if observation continues to be the best management of idiopathic granulomatous mastitis. Surgery 166(6): 1176-1180.

15. Altintoprak F, Kivilcim T, Ozkan OV (2014) Aetiology of idiopathic granulomatous mastitis. World J Clin Cases 2(12): 852-858.

16. Akbulut S, Yilmaz D, Bakir S (2011) Methotrexate in the management of idiopathic granulomatous mastitis: review of 108 published cases and report of four cases. Breast J 17(6): 661-668.

17. Cetin K, Sıkar HE, Göret NE, Rona G, Barıșık NÖ, et al. (2019) Comparison of Topical, Systemic, and Combined Therapy with Steroids on Idiopathic Granulomatous Mastitis: A Prospective Randomized Study. World J Surg 43(11): 2865-2873.

18. Mizrakli T, Velidedeoglu M, Yemisen M, Birgul M, Fahrettin K, et al. (2015) Corticosteroid treatment in the management of idiopathic granulomatous mastitis to avoid unnecessary surgery. Surg Today 45(4): 457-465.

19. Deng JQ, Yu L, Yang Y, Feng XJ, Sun J, et al. (2017) Steroids administered after vacuum-assisted biopsy in the management of idiopathic granulomatous mastitis. J Clin Pathol 70(10): 827-831.

20. Goulabchand R, Perrochia H, Aubert-Bringer E, Do Trinh P, Guilpain $\mathrm{P}$, et al. (2020) Idiopathic granulomatous mastitis responding to oral prednisone. Breast J 26(2): 281-283.

21. Goldberg J, Baute L, Storey L, Park P (2000) Granulomatous mastitis in pregnancy. Obstet Gynecol 96(5 Pt 2): 813-815. 


\section{Your next submission with Juniper Publishers} will reach you the below assets

- Quality Editorial service

- Swift Peer Review

- Reprints availability

- E-prints Service

- Manuscript Podcast for convenient understanding

- Global attainment for your research

- Manuscript accessibility in different formats

( Pdf, E-pub, Full Tsext, Audio)

- Unceasing customer service

Track the below URL for one-step submission https://juniperpublishers.com/online-submission.php 